\title{
Equilibrium Constant, Concentrations Study of p-Aminobenzoic Acid in Anionic, Cationic and Non-ionic Surfactants
}

\author{
Albert Antony Muthu Prabhu ${ }^{1, *}$, Narayanasamy Rajendiran ${ }^{2}$, Kasi Sathiyaseelan ${ }^{1}$ \\ ${ }^{1}$ Department of PG Chemistry, Aditanar College of Arts and Science, Tiruchendur, India \\ ${ }^{2}$ Department of Chemistry, Annamalai University, Annamalainagar, India
}

Email address:

antonyphdchem@yahoo.com (A. A. M. Prabhu)

${ }^{*}$ Corresponding author

\section{To cite this article:}

Albert Antony Muthu Prabhu, Narayanasamy Rajendiran, Kasi Sathiyaseelan. Equilibrium Constant, Concentrations Study of $p$ Aminobenzoic Acid in Anionic, Cationic and Non-ionic Surfactants. Science Journal of Analytical Chemistry. Vol. 9, No. 3, 2021, pp. 68-76. doi: $10.11648 /$ j.sjac.20210903.12

Received: August 18, 2021; Accepted: September 13, 2021; Published: October 12, 2021

\begin{abstract}
ABA})$ was used as a probe to study the equilibrium constant in ground and excited state and concentrations of anionic surfactant, cationic surfactant and non-ionic surfactant. The absorption and fluorescence spectral characteristics of $p \mathrm{ABA}$ were reported in aqueous micellar environments (SDS, CTAB and TW 80) at different $p \mathrm{Hs}$. The neutral and monoanion form of $p \mathrm{ABA}$ is present at both $p \mathrm{Hs} 3$ and 7 respectively. The blue shifted fluorescence maximum was accompanied by a great raise in intensity at $p \mathrm{H} 3$ whereas at $p \mathrm{H} 7$ the emission intensity a little increase with increasing micelles concentration. In the acidic medium, the $\mathrm{CMC}$ value is increased when compared to neutral solution. The strength of interaction between pABA-micelles at both $\mathrm{pHs}$ was estimated using Hirose - Sepulveda equation and these calculated values was further used to estimate the free energy change of the interaction from the fluorescence data. These results from the absorption and fluorescence spectra of pABA well explained that the nature of force and location of probe in ionic micelles and non-ionic micelles at different $\mathrm{pHs}$.
\end{abstract}

Keywords: $p$-Aminobenzoic Acid, Ionic and Non-ionic Micelles, Binding Site

\section{Introduction}

The interaction of surfactants with various charges such as anionic, cationic, non-ionic and many organic neutral molecules has been investigated by the changes in the absorption and fluorescence spectrum [1-3]. These spectral changes after addition of organic molecules were moving into the core or surface of the micelles in the aqueous medium to change the various parameters. Surfactant-induced changes in absorption and fluorescence spectrum have been used to highlight various factors that contribute towards the growth of surfactant-organic molecules aggregates [4-7]. The binding behaviour of these aggregates provides the key to manufacturing of nanostructure materials $[8,9]$.

The acid-base equilibria for many organic molecules having functional groups such as $-\mathrm{OH},-\mathrm{NH}_{2},-\mathrm{COOH},-\mathrm{N}=\mathrm{N}$ etc could be altered in micellar medium. This alteration could be explained the perturbation of the acid-base equilibria by the electrostatic field effect of the charged interface. Santos et al [10] has been studied the binding of chloroquine species to ionic micelles of opposite charges in different $\mathrm{pH}$. Then the interaction of 6-methoxyquinoline with sodium dodecylsulfate micelles was investigated using the spectral techniques at different $\mathrm{pH}$ [11]. Benzophenanthridine alkaloids [12, 13] namely Chelerythrine and Sanguinarine were structurally modified in sodium dodecylsulfate micelles with fluorescence modification at low and high $\mathrm{pH}$.

Crystallographic, spectral and computational study of paminobenzoicacid was investigated with $\alpha$-cyclodextrin [14], $\beta$-cyclodextrin $[15,16]$ and hydroxypropyl- $\beta$-cyclodextrin $[17,18]$ to form stable inclusion complex in aqueous solution and solid phase. Volumetric and calorimetric studies were also reported the inclusion complex formation of cyclodextrins with aminobenzoic acids [19]. Further the 
supramolecular recognition behaviour of sulfonatocalix [6] arene with amino benzoic acid isomers were studied by fluorescence and nuclearmagnetic resonance spectroscopy [20]. The influence of biologically active monovalent cations such as $\mathrm{Na}^{+}$and $\mathrm{K}^{+}$on $\alpha$-cyclodextrin with pABA was studied at different $\mathrm{pH}$ and temperature of $298.15 \mathrm{~K}$ by ${ }^{1} \mathrm{H}$ NMR and calorimetry methods [21].

In the last few years, large number of organic molecules has been investigated in different solvents with various polarity, varying hydrogen ion concentration and $\beta$ cyclodextrin, 2-hydroxypropyl- $\beta$-cyclodextrin on the spectral characteristics of different fluorophores and computational methods [22-24]. The existence of various species such as dication, monocation, neutral, monoanion and dianion in many organic molecules at different $p H s$ were involved in the ground and excited states to study the prototropic reaction in aqueous and $\beta$-cyclodextrin medium $[25,26]$. This prototropic study of organic molecules has been further extended to non-aqueous environment in anionic sodium dodecylsulfate (SDS), cationic cetyltrimethylammonium bromide (CTAB) and neutral polyoxyethylene sorbitanmonooleate (Tween 80) with addition of p-aminobenzoic acid. The presence of functional groups $\left(-\mathrm{NH}_{2},-\mathrm{COOH}\right)$ in pABA makes them $\mathrm{pH}$ sensitive and better fluorescent probe.
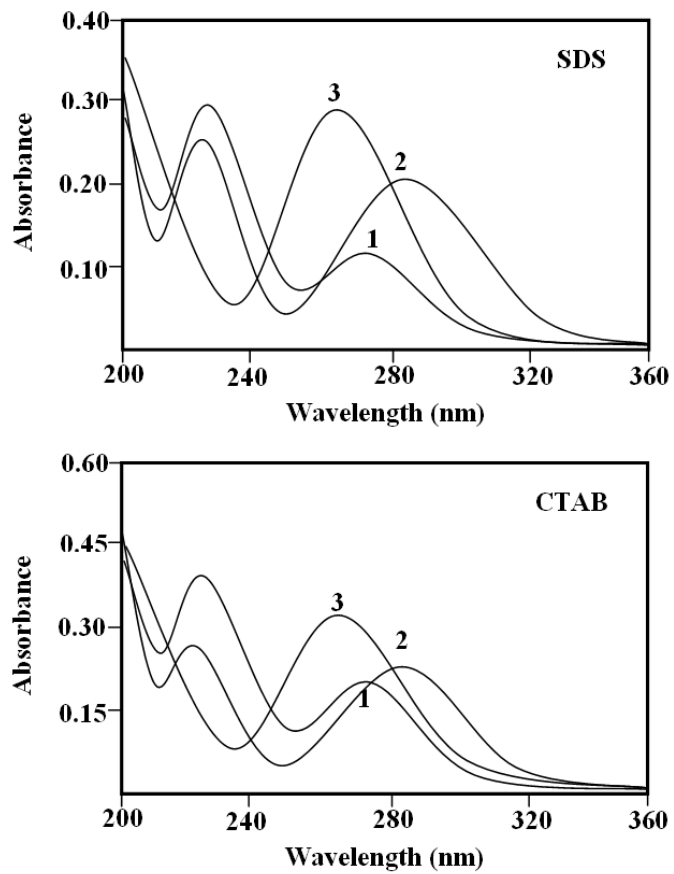

\section{Experimental}

p-Aminobenzoic acid (pABA), Sodium dodecylsulphate (SDS), Cetyltrimethylammonium bromide (CTAB) and Polyoxyethylene sorbitan-monoleate (Tween 80) were obtained from Sigma-Aldrich. Analytical grade $\mathrm{H}_{2} \mathrm{SO}_{4}$, $\mathrm{NaOH}$ and Triply distilled water were used to prepared acidic and basic solutions.

Absorption spectral measurements were carried out with a Shimadzu UV 1601 PC model UV-Visible spectrophotometer and fluorescence measurements were made by using a Shimadzu spectrofluorimeter model RF-5301PC. The $\mathrm{pH}$ of solutions was adjusted in the range of $1-12$ by adding appropriate amount of $\mathrm{H}_{2} \mathrm{SO}_{4}$ or $\mathrm{NaOH}$. In SDS and CTAB, the $\mathrm{pH}$ was maintained by adding $\mathrm{NaOH}$ and $\mathrm{H}_{2} \mathrm{SO}_{4}$, and no buffers were used in this case. The concentration of pABA used was $1 \times 10^{-5} \mathrm{M}$. The stock solutions were prepared in methanol but the final solutions did not contain more than $1 \%$ methanol. The surfactant concentration was varied within the range $5 \times 10^{-2}$ to $10 \times 10^{-5} \mathrm{M}$ depending on the nature of the used surfactant. Before the spectral measurements we kept the micellar solution for 5-6h generally for better equilibration, even for overnight in some cases.
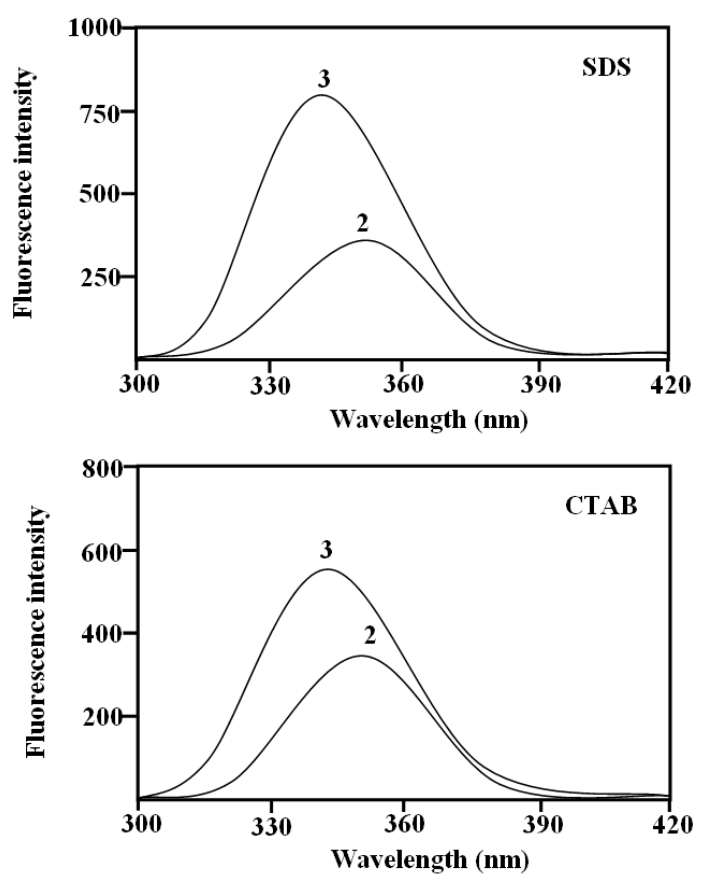

Figure 1. Absorption and fluorescence spectra of prototropic species of pABA in SDS and CTAB. 1. Monocation, 2. Neutral and 3. Monoanion.

Table 1. Absorption and fluorescence maxima of various prototropic species of $\mathrm{PABA}$ in aqueous, SDS and CTAB medium.

\begin{tabular}{|c|c|c|c|c|c|c|}
\hline \multirow{2}{*}{ Species } & \multicolumn{2}{|c|}{ Aqueous } & \multicolumn{2}{|l|}{ SDS } & \multicolumn{2}{|c|}{ CTAB } \\
\hline & $\lambda_{\text {abs }}$ & $\lambda_{\text {flu }}$ & $\lambda_{\text {abs }}$ & $\lambda_{\text {flu }}$ & $\lambda_{\mathrm{abs}}$ & $\lambda_{\text {flu }}$ \\
\hline \multirow{2}{*}{ Monocation } & 271.2 & \multirow{2}{*}{$\mathrm{nf}$} & 271.2 & \multirow{2}{*}{$\mathrm{nf}$} & 271.2 & \multirow{2}{*}{$\mathrm{nf}$} \\
\hline & 226.0 & & 226.0 & & 226.0 & \\
\hline \multirow{2}{*}{ Neutral } & 280.0 & \multirow{2}{*}{350} & 283.0 & \multirow{2}{*}{346} & 285.2 & \multirow{2}{*}{343} \\
\hline & 218.4 & & 220.0 & & 221.0 & \\
\hline Monoanion & 265.4 & 338 & 265.4 & 338 & 265.4 & 337 \\
\hline
\end{tabular}

\section{Results and Discussion}

\subsection{Effect of PH}

The selected amphoteric molecule pABA in this manuscript having $-\mathrm{COOH}$ and $-\mathrm{NH}_{2}$ groups at $4^{\text {th }}$ position in the aromatic ring. The absorption and emission spectra of $\mathrm{pABA}$ at different acid and base concentrations have been 
studied in the aqueous solutions of SDS and CTAB. Figure 1 illustrates the effect of acid and base concentration on the absorption and emission spectra of pABA in $0.05 \mathrm{M}$ SDS and $0.01 \mathrm{M}$ CTAB. The absorption and emission maximum in different $\mathrm{pHs}$ were shown in Table 1. Five prototropic species of pABA such as dication (DC), monocation (MC), neutral $(\mathrm{N})$, monoanion (MA) and dianion (DA) were observed in the aqueous solution. The absorption and emission maximum of all protropic species for pABA in aqueous solution were reported in the earlier literature [27]. But in the case of micellar environment, these species whether or not formed in the ground and excited states, but earlier references reported these types of ionic species were formed in SDS, CTAB and TW 80 micelles. From the comparison of absorption and emission maximum in aqueous solution, the monocation, neutral, monoanion of pABA was appeared at $271 \mathrm{~nm} \&$ nonfluorescence, $280 \& 350 \mathrm{~nm}$ and $265 \& 338 \mathrm{~nm}$ in the micellar environment. At a small hydrogen ion concentrations ( $\mathrm{pH} 1)$, the monocation of $\mathrm{pABA}$ was formed in SDS and CTAB medium from the protonation takes place at 4-position of amino group without changing in absorption and emission maximum compared aqueous solution. Then the hydrogen ion concentrations at $\mathrm{pH}$ range of 2.0 to 3.5 in micellar environment the absorption maximum was moving to the red shift and blue shift in emission maximum for neutral pABA. At neutral $\mathrm{pH}$, the monoanion of pABA was formed without change in the absorption and emission maximum in the micellar environment, it is due to deprotonation from the carboxyl group.

The ground state $\mathrm{pKa}$ of monocation - neutral and neutral monoanion equilibria have been calculated using Henderson equation [28] in aqueous, SDS, CTAB solution according to equation (1) and the equilibria was shown in Figure 2.

$$
\mathrm{pKa}=p H+\log \frac{\mathrm{C}_{1}}{\mathrm{C}_{2}}
$$

The excited state acidity constant of this neutral monoanion equilibrium, $\mathrm{pK}_{\mathrm{a}}{ }^{*}$ has been calculated using Förster cycle [29] according to equation (2):

$$
\mathrm{pKa}-\mathrm{pK}^{*} \mathrm{a}=\frac{\mathrm{E}_{\mathrm{HX}}-\mathrm{E}_{\mathrm{X}}}{2.303 \mathrm{RT}}
$$

where $E_{\mathrm{HX}}$ and $\mathrm{E}_{\mathrm{X}}$ are the energies of electronic transitions for the conjugate acid and base, respectively.

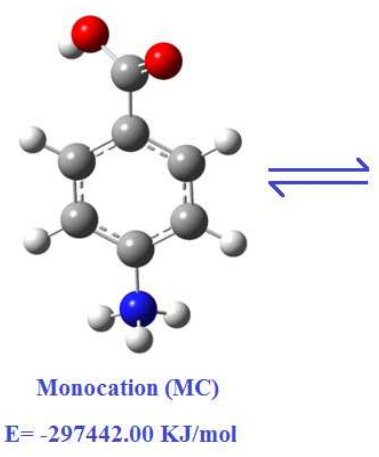

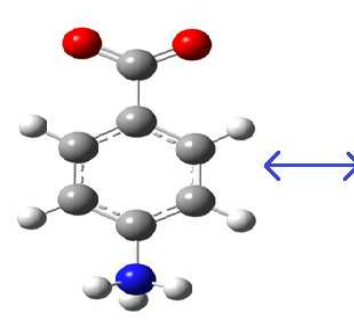

Zwitter ion (Z) $\mathbf{E}=-\mathbf{2 9 7 0 8 4 . 9 2} \mathrm{KJ} / \mathrm{mol}$

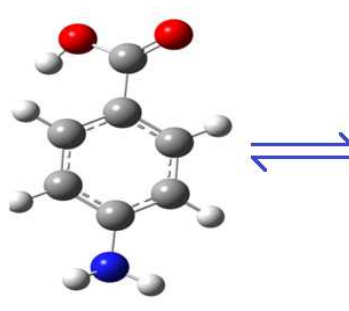

Neutral (N)

$\mathrm{E}=-297143.26 \mathrm{KJ} / \mathrm{mol}$

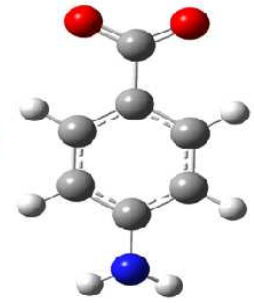

Monoanion (MA)

$E=-296787.25 \mathrm{KJ} / \mathrm{mol}$

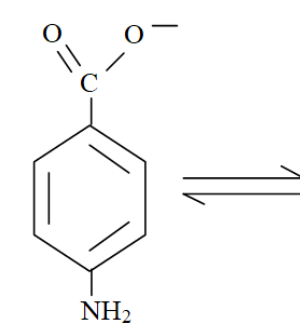

Monocationic

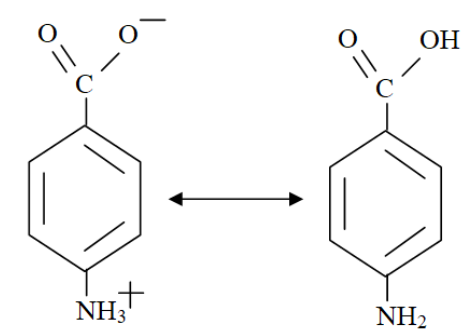

Neutral

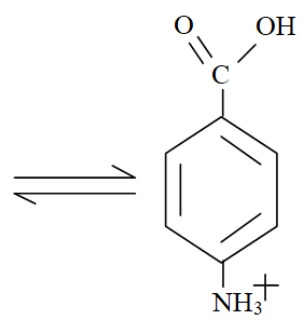

Monoanionic

Figure 2. The conversion of three species of PABA in different $p H$.

The ground and excited state acidity constant ( $\mathrm{pKa}$ and $\left.\mathrm{pKa}^{*}\right)$ values for the equilibrium between monocation neutral and neutral - monoanion of pABA in aqueous, SDS, CTAB media were determined spectrophoto metrically and fluoro metrically methods. The ground state acidity constant for monocation - neutral equilibrium $\left(\mathrm{pK}_{\mathrm{a} 1}\right)$ and neutral monoanion equilibrium $\left(\mathrm{pK}_{\mathrm{a} 2}\right)$ in aqueous solution were calculated as 2.42 and 4.80 , in agreement with the literature $\left(\mathrm{pK}_{\mathrm{a} 1}-2.39\right.$ and $\left.\mathrm{pK}_{\mathrm{a} 2}-4.91\right)$ [30].
In the presence of SDS micelles, $\mathrm{pK}_{\mathrm{a} 1}$ was shifted from 2.42 (aqueous solution) to 2.52, whereas in the presence of CTAB, the $\mathrm{pK}_{\mathrm{a} 1}$ was very slightly reduced to 2.40 from 2.42 when compared to the aqueous medium. Another equilibrium netural - monoanion value was reduced in SDS and that was increased in CTAB micelles. The equilibrium shift was consistent with pABA being located in the charged micelles and electrostatic effects on the surface of micelles may be related to the observed differences in $\mathrm{pKa}$ values. The charge 
distribution in the surface of micelles originates an electric potential that spreads onto the environment, affecting the proton distribution around the micelles.

The positive charge of monocation of $\mathrm{pABA}$ and negative charge of SDS in interfacial layer and the negative charge of monoanion of pABA and positive charge of $\mathrm{CTAB}$ are strongly attracted due to electrostatic attraction between opposite charges. However, $\mathrm{pK}_{\mathrm{a}}{ }^{*}$ value calculated for neutral - monoanion equilibrium was not accurate as the fluorescence band maximum of the monoanion species could not be calculated precisely. The acidity constant for this equilibrium at the excited state $\mathrm{pK}_{\mathrm{a}}{ }^{*}$ has also been calculated using Förster cycle as mentioned above.

\subsection{Effect of Micelles}

In order to demonstrate the effect of acidic and neutral micellar environments on the photophysics of pABA in the presence of anionic surfactant SDS, cationic surfactant CTAB and non-ionic surfactant TW80 with respect to bulk water have been done at two pHs ( 3 and 7 ) of the solution. Absorption and fluorescence spectra of pABA in an aqueous solution with varying concentrations of SDS surfactants at two $\mathrm{pHs}$ have been recorded keeping the concentration of pABA fixed in each case. The concentrations of SDS, CTAB and TW80 taken into account are pre-micellar, micellar and post-micellar concentrations. The absorption and fluorescence spectra of fixed concentrations of pABA $\left(2 \times 10^{-}\right.$ $\left.{ }^{5} \mathrm{M}\right)$ in SDS $\left(2 \times 10^{-3} \mathrm{M}\right.$ to $\left.5 \times 10^{-2} \mathrm{M}\right)$, CTAB $\left(4 \times 10^{-4} \mathrm{M}\right.$ to $\left.1 \times 10^{-2} \mathrm{M}\right)$ and TW80 $\left(2 \times 10^{-5} \mathrm{M}\right.$ to $\left.5 \times 10^{-4} \mathrm{M}\right)$ surfactants of different concentrations in aqueous media at two pHs were shown in figures 3 and 4 . Table 2 describes the absorption and fluorescence maxima and other calculated parameters of pABA in SDS, CTAB and TW 80.
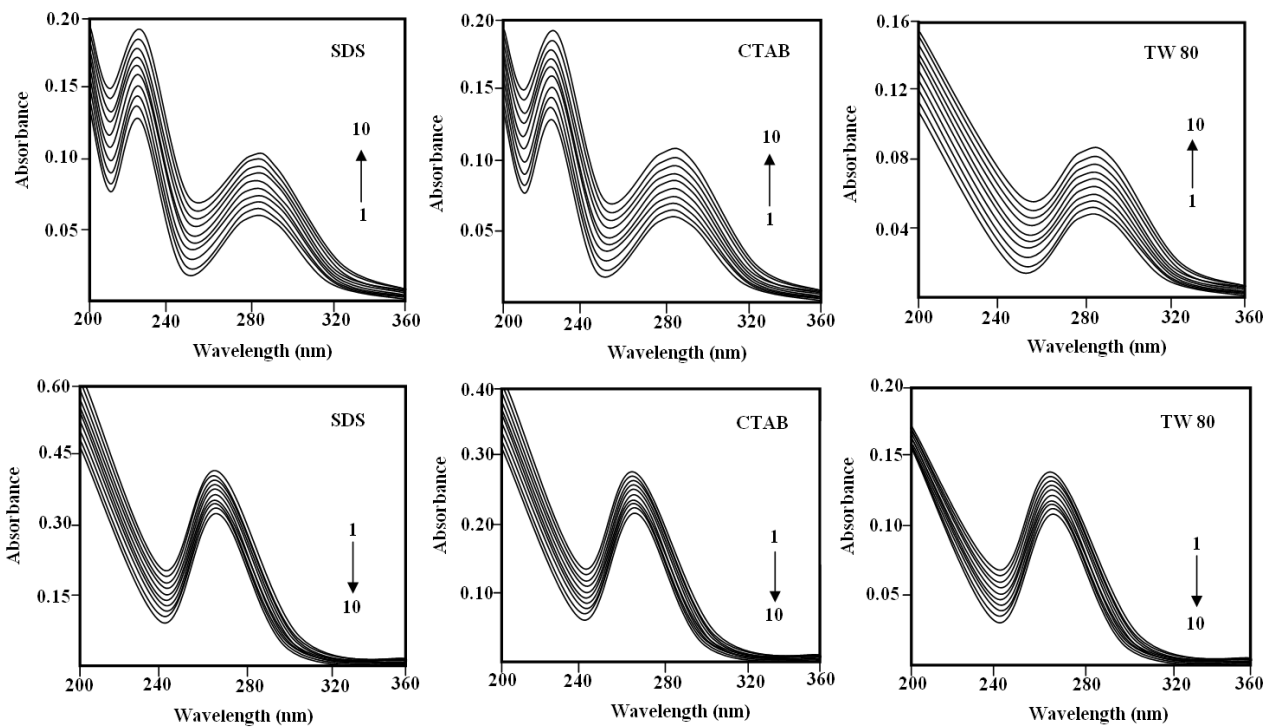

Figure 3. Absorption spectra of pABA in different SDS, CTAB and TW 80 concentrations (mol dm ${ }^{-3}$ ) at pH 3.0 and pH 7.0 .
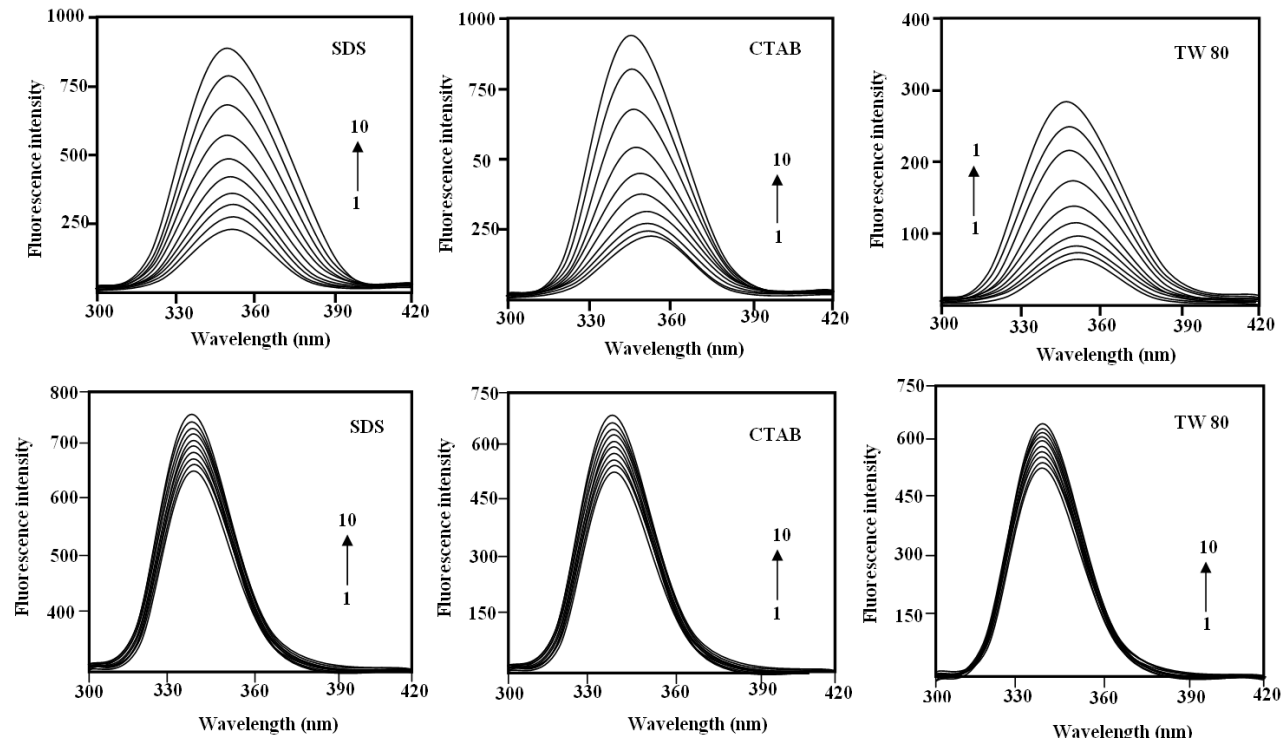

Figure 4. Fluorescence spectra of $4 A B A$ in different $S D S, C T A B$ and $T W 80$ concentrations (mol dm ${ }^{-3}$ ) at $\mathrm{pH} 3.0$ and $\mathrm{pH} 7.0$. 
Table 2. Absorption, fluorescence maxima, CMC, binding constant and free energy of pABA in SDS, CTAB and TW 80 at two $p H s$.

\begin{tabular}{|c|c|c|c|c|c|c|c|}
\hline S.No. & Medium & $\lambda_{\max }$ & $\log \varepsilon$ & $\lambda_{\text {flu }}$ & CMC & Binding Constant & $\Delta G(-v e) \mathrm{KJ} / \mathrm{mol}$ \\
\hline 1 & Water ( $p \mathrm{H} 3.0)$ & 280 & 3.81 & 349 & & & \\
\hline 2 & Water $(p \mathrm{H} 7.0)$ & $\begin{array}{l}224 \\
265\end{array}$ & $\begin{array}{l}3.97 \\
4.14\end{array}$ & 338 & & & \\
\hline 3 & $\operatorname{SDS}(p \mathrm{H} 3.0)$ & $\begin{array}{l}282 \\
226\end{array}$ & $\begin{array}{l}3.48 \\
4.00\end{array}$ & 348 & $16 \times 10^{-3}$ & 80 & -11.03 \\
\hline 4 & $\operatorname{SDS}(p H 7.0)$ & 265 & 4.08 & 339 & $10 \times 10^{-3}$ & 37 & -9.09 \\
\hline 5 & CTAB $(p \mathrm{H} 3.0)$ & $\begin{array}{l}287 \\
226\end{array}$ & $\begin{array}{l}4.00 \\
3.96\end{array}$ & 343 & $1.8 \times 10^{-3}$ & 485 & -15.57 \\
\hline 6 & СТAВ ( $p$ H 7.0) & 267 & 4.04 & 337 & $1.1 \times 10^{-3}$ & 320 & -14.54 \\
\hline 7 & TW $80(p \mathrm{H} 3.0)$ & 289 & 3.71 & 346 & $13 \times 10^{-5}$ & 7820 & -22.57 \\
\hline 8 & TW $80(p H 7.0)$ & 266 & 3.93 & 338 & $9 \times 10^{-5}$ & 5630 & -21.77 \\
\hline
\end{tabular}
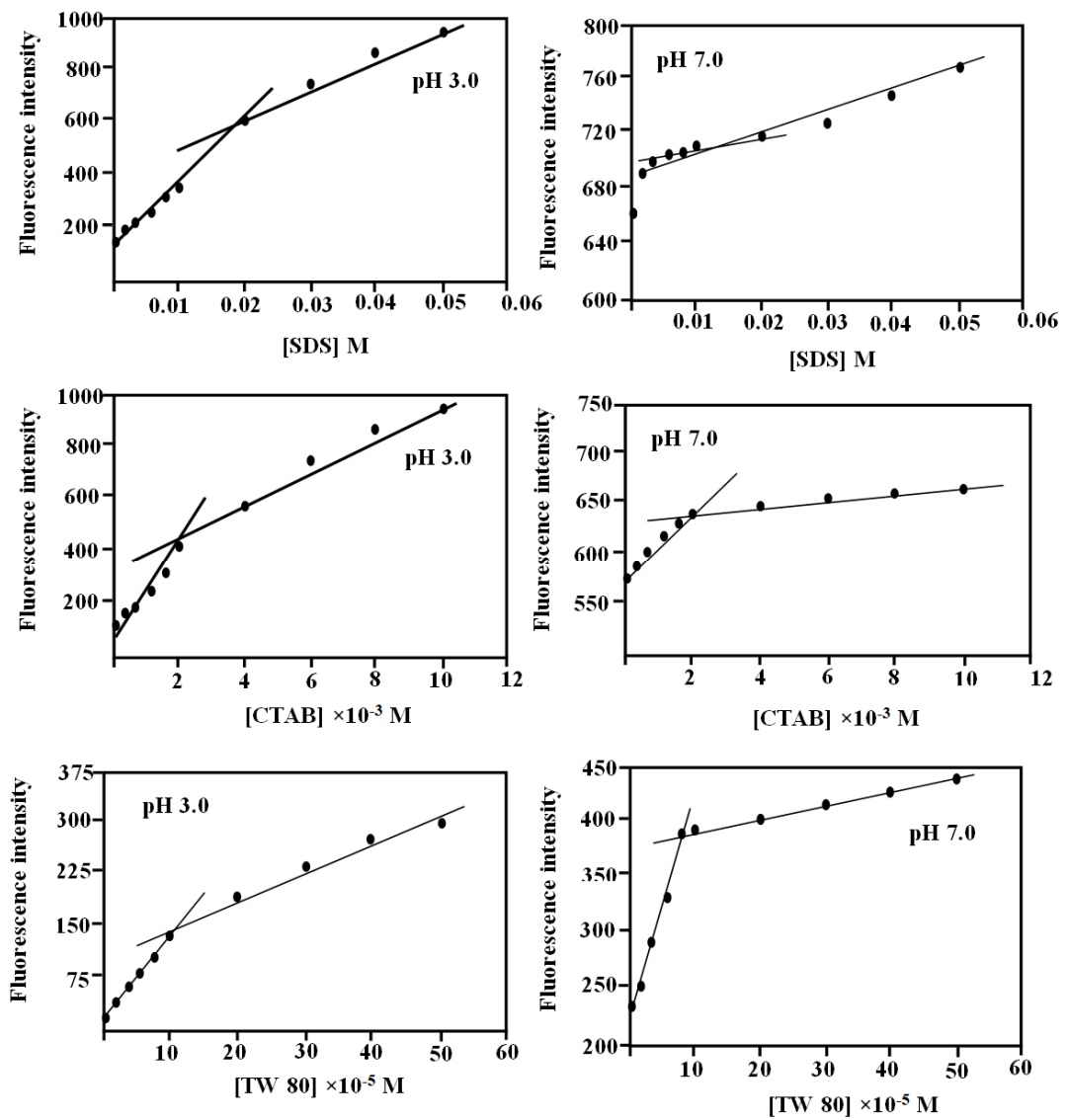

Figure 5. Plot of fluorescence intensity versus concentration of SDS, CTAB and TW 80.

On addition of the surfactants to the aqueous solution of pABA at $\mathrm{pH} 3$ leads to a red shift $\left(\lambda_{\mathrm{abs}}-280 \mathrm{~nm}\right.$ to $289 \mathrm{~nm}$ with respect to micelles) in the absorption maximum whereas the absorption maximum has no significant shift at $\mathrm{pH} 7$ in all the micelles. The molar extinction coefficient of the longer wavelength absorption band was increased with increasing concentration of all micelles. At $\mathrm{pH} \mathrm{7,} \mathrm{the} \mathrm{molar}$ extinction coefficient was decreased with increasing concentration of all micelles. These results in absorption spectrum and absorbance revealto change the microenvironment of neutral and monoanion species of pABA.

Figure 4 depict the fluorescence spectra of pABA in aqueous solution of SDS, CTAB and TW 80 as a function of surfactant concentrations. Addition of surfactants to an aqueous solution of $\mathrm{pABA}$ at both $\mathrm{pHS}$ resulted in the variety of fluorescence signals. At $\mathrm{pH} 3$ gradual additions of surfactants (SDS, CTAB and TW 80) is associated with a slight blue shift of the emission maximum along with an enhancement of the emission intensity. The monoanion fluorescence maximum of pABA not much affected with the addition of the surfactants. It was clear that the fluorescence enhancement in acidic media was stronger than that in neutral media. The above observations reflect the micro environments around the fluorophore in the micellar solutions being quite different from those in the bulk aqueous phase at two pHs. A blue shift and enhanced emission intensity in the neutral emission spectrum within the micellar solutions further suggests that the polarity of the micellar environments is less than the polarity of the bulk water $[31,32]$. 

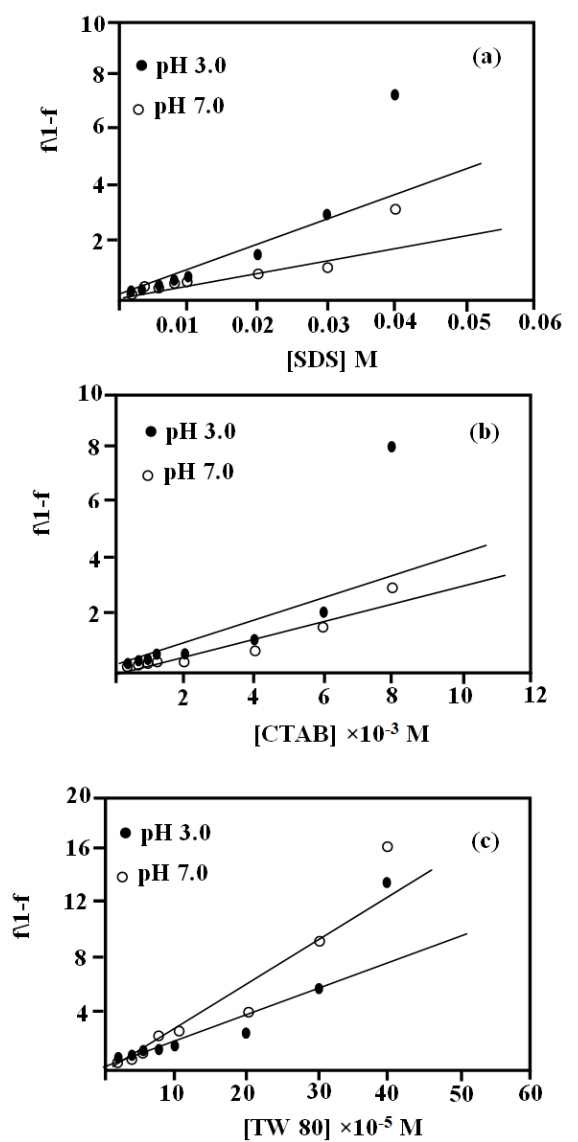

Figure 6. Plot of $f(1-f$ against surfactant concentration (a) SDS, (b) CTAB and (c) $T W 80$.

\subsection{Critical Micelle Concentration}

The changes in fluorescence maximum and intensity with the addition of surfactants can be attributed to the passage of pABA molecule from a highly polar aqueous medium to a relatively nonpolar micellar environment. At lower surfactant concentrations the change in fluorescence response was not high. However, after a certain micellar concentration, fluorescence intensity shows a marked change. These sharp break points are conventionally assigned to critical micelle concentration. Typical plots of fluorescence intensity variation with surfactant concentration are shown in Figure 5 and the critical micellar concentrations obtained from the inflexion points for the micelles are complied in Table 2. Generally, the CMC value was decreased with increase in $\mathrm{pH}$. In the acidic medium, the CMC value was higher in all the micelles when compared to the neutral solution.

\section{4. pABA-Micelle Binding}

The binding constant (Ks) to all the micelles have been determined using the Hirose - Sepulveda equation [33].

$$
\frac{\mathrm{f}}{1-\mathrm{f}}=K s[D] t-K s C M C
$$

where $[D]_{t}$ is the total surfactant concentration and ' $\mathrm{f}$ ' is the fraction of the pABA bound to micelle.

$$
f=\frac{I-I_{w}}{I_{m}-I_{w}}
$$

where $I, I_{m}$, and $I_{w}$ are the absorption or fluorescence intensities at intermediate micellar concentration, at complete micellization and in the absence of micelles, respectively. $\mathrm{K}$ is being the binding constant. The values of ' $\mathrm{f}$ ' used vary between 0.2 to 0.8 in order to have the minimized error. A plot of $\mathrm{f} / 1-\mathrm{f}$ versus $[\mathrm{D}]_{\mathrm{t}}$ is a straight line (Figure 6). The binding constant values have been determined from the slopes of the individual plots.

The thermodynamic parameter $\Delta \mathrm{G}$ which is an indicator of a tendency of the binding of pABA to micelles, was calculated using the following equation:

$$
\Delta \mathrm{G}=-\mathrm{RT} \ln \mathrm{K}
$$

The interactions of pABA with micelles are exothermic. As seen in Table 2, the standard free energy change values for pABA in TW 80 are bigger than in CTAB and SDS. Therefore, pABA interacts with non-ionic micelles more easily and stronger than with cationic and anionic micelles in both pHs.

The photophysical characteristics of p-aminobenzoic acid have been well studied in various solvents and buffer solutions [27]. The solvent study revealed that ICT emission is not present in pABA. The absorption and emission spectrum in the aqueous solution at $\mathrm{pH} 7$ is largely blue shifted than the other solvents. Acidic $\mathrm{pH}$ did not affect the appearance of the spectrum except for a red shift. This effect of the $\mathrm{pH}$ on the absorption and emission spectrum indicates that the carboxyl group was ionized at $\mathrm{pH} 7$.

The effect of SDS, CTAB and TW 80 on the fluorescence spectra of pABA more sensitive to the corresponding effect of the absorption spectra. With an increasing the micelles concentration, the fluorescence intensities of pABAwas increased in all the micelles at both pHs. Therefore, pABA tend to move towards micellar environment. In the absence and presence of micelles, a single emission was observed at both pHs. In $\mathrm{pH} 3$, blue shift emission maximum was observed in all the micelles, while in $\mathrm{pH} 7$ there is no significant shift noticed with an increasing the micelle concentration. The molecular species of pABA showing the remarkable rise in fluorescence intensity with all the surfactants. Figure 7 shows the probable location of neutral and monoanion species of pABA in SDS and CTAB. In micelles, the blue shifted emission at $\mathrm{pH} 3$ suggests that the amino group is present in the non-polar environment of micelles. These observations indicate that all the micelles provide pABA with less polar environments than water. Thus, the blue shifted emission should be the normal emission originated from the initially excited state of the neutral species. At $\mathrm{pH} 7$ there is no significant shift emission maxima along with a small enhancement in the micellar medium clearly established that the anionic species of pABA may be present in the aqueous medium. 


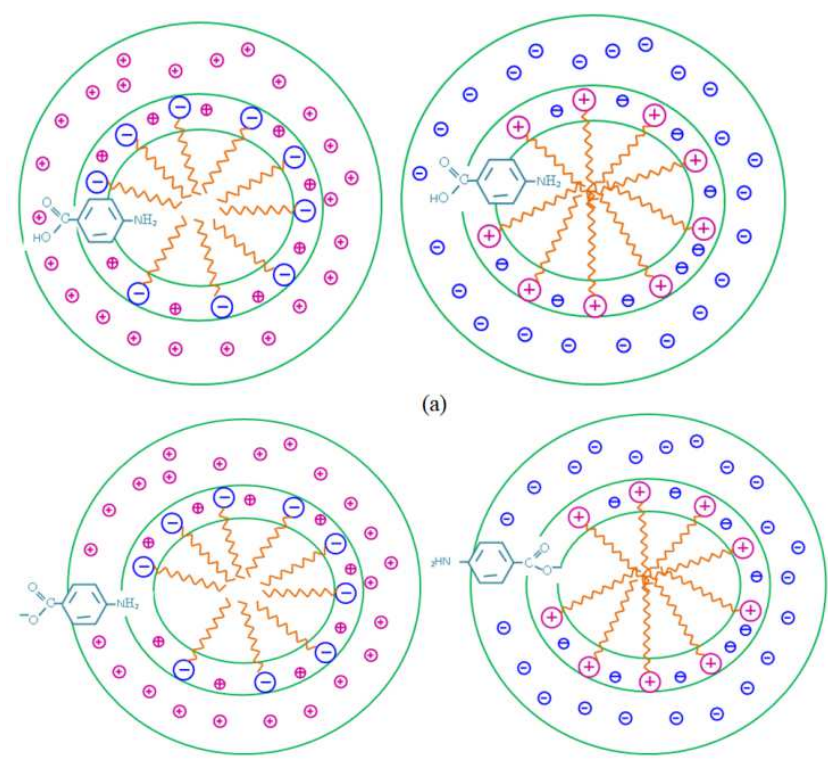

(b)

Figure 7. The probable location of neutral and monoanion of $p A B A$ (a) neutral and (b) monoanion in SDS and CTAB.

The trend observed in the spectral characteristics of the monoanion was varied to that for the neutral pABA. This is expected because the monoanion will be present in the interfacial region and enough water molecules will be present at the interface to solvate them. From the above discussion and the data of Table 2, it can be qualitatively concluded that pABA is transferred from a highly polar and protic phase to a less polar and protic site in the micellar phase. The strength of binding interaction favourable for neutral species in all the micelles indicates that the micelles provide a hydrophobic environment to the probe. This result about binding strength and hydrophobic environment gives more interaction for neutral comparing to anionic species. In case of SDS, the binding constant of monoanion species is lower than its neutral molecule because electrostatic repulsion between the anionic sulphate groups and the anionic species of carboxylic acid unfavorable the binding process. Electrostatic interactions cause generally a red shift of absorption and emission as the polarity of the solvent increases [34].

In SDS and CTAB micelles both the hydrophobic and electrostatic interaction comes into play on the pABA whereas in TW 80 only the hydrophobic force was present due to the absence of columbic interaction. At $\mathrm{pH} 3$ the blue shifted emission maximum is responsible for hydrophobic interaction between pABA and micelles. Therefore the hydrophobic interaction is predominant over the electrostatic interaction. The enhancement of the neutral emission intensity in SDS and CTAB is much higher than those observed in TW 80. In the absence of electrostatic interaction, pABA would go to the core of the micelles. At $\mathrm{pH} 7$ the anion emission intensity of pABA is slightly increased with increasing concentration of micelles. Therefore the anion species of pABA will be located in the aqueous medium. The electrostatic field in the ionic micelles would restrict pABA to go to the micellar region. The neutral species of pABA is greater binding than anionic species of pABA. The interaction of organic molecules in micelles was involved by the nature of the force and location of the probe. This one clearly explains with the support from the calculation of binding constant for all micelles in the presence of probe. This parameter in this medium is an important parameter based on the fluorescence data.

The amino group of neutral form of pABA is located in the interior of micellar and the carboxylic acid group is present at the micelle-water interface in both the micelles. The anion form is present in the micelle-water interface. The red shifted absorption in SDS and CTAB can be due to the H-bonding effect of the pABA in micellar environments. The hydrogen bonding can occur between the lone pair electrons on the oxygen atom of the solvent molecule with the hydrogen atom of the carboxylic acid group of pABA. The penetration of the $\mathrm{pABA}$ and its location in micelles depends on the nature of the force and polarity variation with different layers in SDS and $\mathrm{CTAB}$ micelles. Another factor for the interaction between pABA and micelles, the flexibility of aliphatic chain determines the movement of pABA easier towards into the inside of micellar environment. This is more easily to make in CTAB micelles compared to SDS micelles.

\subsection{Polarity of the Micellar Environments}

Quantitatively, the observation of a high value of binding constant for neutral pABA was completely micellized upto a high concentration of micelles. The spectral characteristics of pABA in surfactants are useful for understanding the nature and polarity of the binding sites. In order to estimate the nature of the average binding site in the cationic, anionic and non-ionic micelles, correlation of spectral shifts with different polarity parameters in homogeneous environments was carried. The polarity scales, i.e. Stokes shift versus $\mathrm{E}_{\mathrm{T}}(30)$ polarity parameters [35] have been used to determine the polarity at the binding site of the micelles. It is shown in Figure 8 that the plot of stokes shift versus $\mathrm{E}_{\mathrm{T}}(30)$ shows excellent linear variation. Substitution of corresponding values for SDS, CTAB and TW 80 allowed us to determine polarity values corresponding to solvent polarity parameter $\mathrm{E}_{\mathrm{T}}(30)$ of $58.5,47.5$ and 48.5 respectively. These values are well within the range of some literature [36].

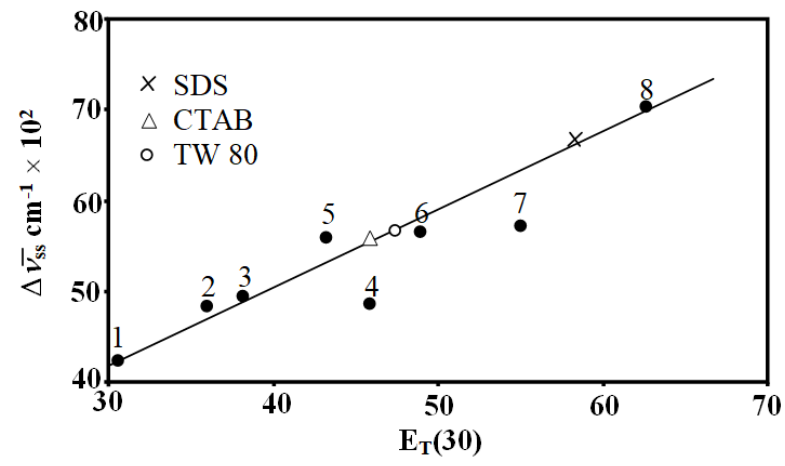

Figure 8. Plot of Stokes shifts ( $\left.\mathrm{cm}^{-1}\right)$ of pABA versus ET(30): 1. cyclohexane, 2. dioxane, 3. ethyl acetate, 4. acetonitrile, 5. t-butyl alcohol, 6. 2-propanol, 7. methanol, 8. water. 


\section{Conclusion}

The prototropic reactions of $\mathrm{pABA}$ was studied in micellar environments $-0.05 \mathrm{M}$ SDS and $0.01 \mathrm{M} \mathrm{CTAB}$ with $\mathrm{pH}$ range from 1 to 14 . The monocation - neutral and neutral monoanion equilibrium constant values were shifted in micellar environments compared to aqueous medium. With increasing of SDS, CTAB and TW 80, the absorption and emission data was considerably changed at two $\mathrm{pHs}$ due to the changing microenvironment of neutral and monoanion species of pABA. Binding constant and solvent polarity parameter ET(30) was calculated from the emission data. Finally the nature of the force and the probable location of neutral and monoanion species of pABA was predicted in SDS, CTAB and TW 80 micellar environments.

\section{References}

[1] S. Sekhar Bag, Rajen Kundu, Sensing of Micellar Microenvironment with Dual Fluorescent Probe, Triazolylpyrene (TNDMBPy), J. Fluoresc., 23 (2013) 929938.

[2] A. Samanta, B. K. Paul, N. Guchhait, Modulation of Intramolecular Charge Transfer Emission Inside Micelles: A Fluorescence Probe for StudyingMicroenvironment of Micellar Assemblies, J. Fluoresc., 22 (2012) 289-301.

[3] A. Asefa, A. K. Singh, A Fluorescence Study of Novel Styrylindoles in Homogenous and Microhetrogeneous Media, J. Fluoresc., 19 (2009) 921-930.

[4] S. Gokturk, M. Tuncay, Spectral studies of safranin-O in different surfactant solutions, Spectrochim. Acta A, 59 (2003) $1857-1866$.

[5] S. De, A. Girigoswami, A. K. Mandal, Energy transfer - a tool for probing micellar media, Spectrochim. Acta A, 59 (2003) 2487-2496.

[6] J. Yang, Interaction of surfactants and aminoindophenol dye, J. Colloid Interface Sci., 274 (2004) 237-243.

[7] H. Akbas, C. Kartal, Spectrophotometric studies of anionic dye - cationic surfactant interactions in mixture of cationic and nonionic surfactants, Spectrochim. Acta A, 61 (2005) 961966.

[8] C. F. J. Faul, M. Antonietti, Facile synthesis of optically functional, highly organized nanostructures: Dye-surfactant complexes, Chem. Eur. J., 8 (2002) 2764-2768.

[9] Y. Guan, M. Antoinietti, C. F. J. Faul, Ionic self-assembly of dye-surfactant complexes: Influence of tail lengths and dye architecture on the phase morphology, Langmuir, 18 (2002) 5939-5945.

[10] M. S. Santos, M. P. F. de Morais Del Lama, A. S. Ito, R. M. Z. Georgetto Naal, Binding of chloroquine to ionic micelles: Effect of $\mathrm{pH}$ and micellar surface charge, J. Lumines., 147 (2014) 49-58.

[11] Y. T. Varma, D. D. Pant, Interaction of 6-methoxyquinoline with anionic sodium dodecylsulfate micelles: Photophysics and rotational relaxation dynamics at different $p \mathrm{H}$, Spectrochim. Acta A, 158 (2016) 9-17.
[12] A. B. Pradhan, S. Bhuiya, L. Haque, R. Tiwari, S. Das, Micelle assisted structural conversion with fluorescence modulation of benzophenanthridine alkaloids, Spectrochim. Acta A, 170 (2017) 89-96.

[13] S. Satpathi, K. Gavvala, P. Hazra, Fluorescence switching of sanguinarine in micellar environments, Phys. Chem. Chem. Phys., 17 (2015) 20725-20732.

[14] K. Elasaad, B. Norberg, J. Wouters, Crystallographic, UV spectroscopic and computational studies of the inclusion complex of $\alpha$-cyclodextrin with p-aminobenzoic acid, Supramolecular Chemistry, 24 (2012) 312-324.

[15] N. V. Roik, L. A. Belyakova, Thermodynamic, IR spectral and $\mathrm{X}$-ray diffraction studies of the " $\beta$-cyclodextrin-paraaminobenzoic acid" inclusion complex, J. Incl. Phenom. Macrocycl. Chem., 69 (2011) 315-319.

[16] I. V. Terekhova, R. S. Kumeev, G. A. Alper, Inclusion complex formation of $\alpha$ - and $\beta$-cyclodextrins with aminobenzoic acids in aqueous solution studied by ${ }^{1} \mathrm{H}$ NMR, J. Incl. Phenom. Macrocycl. Chem., 59 (2007) 301-306.

[17] S. Shaomin, Y. Yu, P. Jinghao, Study on molecular recognition of para-aminobenzoic acid species by $\alpha-, \beta-$ and hydroxypropyl- $\beta$-cyclodextrin, Analytica. Chimica. Acta, 458 (2002) 305-310.

[18] I. Terekhova, R. Kumeev, G. Alper, S. Chakraborty, H. PerezSanchez, E. Nunez-Delicadoc, Molecular recognition of aromatic carboxylic acids by hydroxypropyl- $\beta$-cyclodextrin: experimental and theoretical evidence, $R S C A d v$., 6 (2016) 49567.

[19] I. V. Terekhova, Volumetric and calorimetric study on complex formation of cyclodextrins with aminobenzoic acids, Mendeleev Commun., 19 (2009) 110-112.

[20] C. Zhang, J. Chao, Y. Zhang, G. Zhang, S. Shuang, Investigation on the supramolecular recognition behaviour of sulfonatocalixarene with amino benzoic acid isomers, Phys. Chem. Liquids, 54 (2016) 27-36.

[21] A. Romanova, E. Chibunova, R. Kumeev, M. Fedorov, I. Terekhova, $\alpha$-cyclodextrin/aminobenzoic acid binding in salt solutions at different $\mathrm{pH}$ : Dependence on guest structure, International Journal of Biological Macromolecules, 57 (2013) 255-258.

[22] A. Antony Muthu Prabhu, R. K. Sankaranarayanan, G. Venkatesh, N. Rajendiran, Dual Fluorescence of Fast Blue RR and Fast Violet B: Effects of Solvents and Cyclodextrin Complexation, J. Phys. Chem. B, 116 (2012) 9061-9074.

[23] A. Antony Muthu Prabhu, Madi Fatiha, Nouar Leila, T. Anantha Raj, I. Navarro-Gonzalez, M. J. Periago, M. J. YanezGascon, H. Perez-Sanchez, Investigation of 3D Contour Map and Intermolecular Interaction of Dopamine with $\beta$ Cyclodextrin and 2-Hydroxypropyl- $\beta$-cyclodextrin, J. Solution Chem., 47 (2018) 409-429.

[24] M. Jude Jenita, A. Antony Muthu Prabhu, N. Rajendiran, Encapsulation of 3,5-dihydroxybenzoic acid and 3,4,5trihydroxybenzoic acid by $\alpha$ - and $\beta$-cyclodextrins: Spectral and theoretical studies, J. Indian Chem. Soc., 91 (2014) 1-20.

[25] A. Antony Muthu Prabhu, S. Siva, R. K. Sankaranarayanan, N. Rajendiran, Intramolecular Proton Transfer Effects on 2,6diaminopyridine, J. Fluoresc., 20 (2010) 43-54. 
[26] S. Siva, G. Venkatesh, A. Antony Muthu Prabhu, R. K. Sankaranarayanan, N. Rajendiran, Absorption and fluorescence spectral characteristics of norepinephrine, epinephrine, isoprenaline, methyl dopa, terbutaline and orciprenaline drugs, Phys. Chem. Liquids, 50 (2012) 434-452.

[27] T. Stalin, B. Shanthi, P. Vasantha Rani, N. Rajendiran, Solvatochromism, Prototropism and Complexation of paraaminobenzoic Acid, J. Inclu. Phenom. and Macrocyclic. Chem. 55 (2006) 21-29.

[28] P. V. Jaiswal, V. S. Ijeri, A. K. Srivastava, Effect of surfactants on the dissociation constants of ascorbic and maleic acids, Colloids and Surfaces B: Biointerfaces, 46 (2005) 45-51.

[29] T. Stalin, G. Sivakumar, B. Shanthi, A. Sekar, N. Rajendiran, Photophysical behaviour of 4-hydroxy-3,5-dimethoxybenzoic acid in different solvents, $p \mathrm{H}$ and $\beta$-cyclodextrin, $J$. Photochem. Photobiol. A Chem., 177 (2006) 144-155.

[30] N. O. Mchedlov-Petrossyan, T. A. Cheipesh, N. A. Vodolazkaya, Acid-base dissociation and tautomerism of two aminofluorescein dyes in Solution, Journal of Molecular Liquids., 225 (2017) 696-705.

[31] A. Mallick, S. Maiti, B. Haldar, P. Purkayastha, N. Chattopadhyay, Photophysics of 3-acety-4-oxo-6,7-dihydro$12 \mathrm{H}$ indolo-[2,3-a]quinolizine: emission from two states, Chem. Phys. Lett., 371 (2003) 688-693.
[32] A. Mallick, B. Haldar, S. Maiti, N. Chattopadhyay, Constrainedphotophysics of 3-acetyl-4-oxo-6,7-dihydro-12H indolo-[2,3-a]quinolizine in micellar environments: a spectrofluorometric study, J. Colloid and Interface Sci., 278 (2004) 215-223.

[33] C. Hirose, L. Sepulveda, Transfer free energies of p-alkylsubstituted benzene derivatives, benzene and toluene from water to cationic and anionic micelles and to n-heptane, $J$. Phys. Chem., 85 (1981) 3689-3694.

[34] H. R. Park, B. Mayer, P. Wolschann, G. Kohler, Excited state proton transfer of 2-naphthol inclusion complexes with cyclodextrins, J. Phys. Chem., 98 (1994) 6158-6166.

[35] M. Kosower, A. Dodink, K. Tanizawa, M. Ottolenght, N. Orbach, Intramolecular dono-acceptor systems. Radiative and nonradiative processes for the excited states of 2-Narylamino-6-naphthalenesulfonates, J. Am. Chem. Soc., 97 (1975) 2167-2178.

[36] T. Sanjoy Singh, S. Mitra, Fluorescence behavior of intramolecular charge transfer probe in anionic, cationic and nonionic micelles, J. colloid Interface Sci., 311 (2007) 128134. 